\title{
Permutation Tableaux and the Dashed Permutation Pattern 32-1
}

\author{
William Y.C. Chen ${ }^{1}$, Lewis H. Liu ${ }^{2}$ \\ ${ }^{1,2}$ Center for Combinatorics, LPMC-TJKLC \\ Nankai University, Tianjin 300071, P.R. China \\ ${ }^{1}$ chen@nankai.edu.cn, ${ }^{2}$ lewis@cf c.nankai.edu.cn
}

Submitted: Dec 25, 2010; Accepted: May 4, 2011; Published: May 16, 2011

Mathematics Subject Classifications: 05A05, 05A19

\begin{abstract}
We give a solution to a problem posed by Corteel and Nadeau concerning permutation tableaux of length $n$ and the number of occurrences of the dashed pattern $32-1$ in permutations on $[n]$. We introduce the inversion number of a permutation tableau. For a permutation tableau $T$ and the permutation $\pi$ obtained from $T$ by the bijection of Corteel and Nadeau, we show that the inversion number of $T$ equals the number of occurrences of the dashed pattern 32-1 in the reverse complement of $\pi$. We also show that permutation tableaux without inversions coincide with L-Bell tableaux introduced by Corteel and Nadeau.
\end{abstract}

\section{Introduction}

Permutation tableaux were introduced by Steingrímsson and Williams [14] in the study of totally positive Grassmannian cells $[11,13,16]$. They are closely related to the PASEP (partially asymmetric exclusion process) model in statistical physics [5, 8, 9, 10]. Permutation tableaux are also in one-to-one correspondence with alternative tableaux introduced by Viennot [15].

A permutation tableau is defined by a Ferrers diagram possibly with empty rows such that the cells are filled with 0's and 1's subject to the following conditions:

(1) Each column contains at least one 1.

(2) There does not exist a 0 with a 1 above (in the same column) and a 1 to the left (in the same row).

The length of a permutation tableau is defined as the number of rows plus the number of columns. A 0 in a permutation tableau is said to be restricted if there is a 1 above. 
Among the restricted 0 's in a row, the rightmost 0 plays a special role, which is called a rightmost restricted 0 . A row is said to be unrestricted if it does not contain any restricted 0. A 1 is called essential if it is either the topmost 1 in a column or the leftmost 1 in a row, see Burstein [2]. A permutation tableau $T$ of length $n$ is labeled by the elements in $[n]=\{1,2, \ldots, n\}$ in increasing order from the top right corner to the bottom left corner. The set $[n]$ is referred to as the label set of $T$. We use $(i, j)$ to denote the cell with row label $i$ and column label $j$.

For example, Figure 1.1 exhibits a permutation tableau of length 11 with an empty row. There are two rightmost restricted 0 's at cells $(5,9)$ and $(8,10)$, and there are four unrestricted rows labeled by $1,2,7$ and 11 .

\begin{tabular}{|c|c|c|c|c|}
\hline 0 & 1 & 0 & 0 & 0 \\
\hline 0 & 1 & 0 & 1 & 1 \\
\hline 0 & 0 & 1 & $5^{4}$ & 3 \\
\hline 1 & 1 & $7^{6}$ & & \\
\hline 0 & 1 & 8 & & \\
\hline
\end{tabular}

Figure 1.1: A permutation tableau.

It is known that the number of permutation tableaux of length $n$ is $n$ !. There are several bijections between permutation tableaux and permutations, see Corteel and Nadeau [7], Steingrímsson and Williams [14]. The second bijection in [14] connects the number of 0 's in a permutation tableau to the total number of occurrences of the dashed patterns 31-2, 21-3 and 3-21. This bijection also yields a relationship between the number of 1's in a permutation tableau and the number of occurrences of the dashed pattern 2-31 in a permutation. In answer to a question of Steingrímsson and Williams [14], Burstein [2] found a classification of zeros in permutation tableaux in connection with the total number of occurrences of the dashed patterns 31-2 and 21-3, and the number of occurrences of the dashed pattern 3-21.

On the other hand, the second bijection of Corteel and Nadeau [7] implies that the number of non-topmost 1's in a permutation tableau equals the number of occurrences of the dashed pattern 31-2 in the corresponding permutation. They raised the problem of finding a statistic on permutation tableaux that has the same distribution as the number of occurrences of the dashed pattern $32-1$ in permutations.

Let us recall the definition of dashed permutation patterns introduced by Babson and Steingrímsson [1]. A dashed pattern is a permutation on $[k]$, where $k \leq n$, that contains dashes indicating that the entries in a permutation on $[n]$ need not occur consecutively. In this notation, a permutation pattern $\sigma=\sigma_{1} \sigma_{2} \cdots \sigma_{k}$ in the usual sense may be rewritten as $\sigma=\sigma_{1}-\sigma_{2}-\cdots-\sigma_{k}$. For example, we say that a permutation $\pi$ on $[n]$ avoids the dashed 
pattern 32-1 if there are no subscripts $i<k$ such that $\pi_{i-1}>\pi_{i}>\pi_{k}$. Claesson and Mansour [4] found explicit formulas for the number of permutations containing exactly $i$ occurrences of a dashed pattern $\sigma$ of length 3 for $i=1,2,3$.

The main idea of this paper is to introduce the inversion number of a permutation tableau. We show that the inversion number of a permutation tableau of length $n$ has the same distribution as the number of occurrences of the dashed pattern $32-1$ in a permutation on $[n]$. To be more specific, for a permutation tableau $T$ and the permutation $\pi$ obtained from $T$ by the first bijection of Corteel and Nadeau, we prove that the inversion number of $T$ equals the number of occurrences of the dashed pattern $32-1$ in the reverse complement of $\pi$. This gives a solution to the problem proposed by Corteel and Nadeau $[7]$.

The inversion number of a permutation tableau is defined based on the order of alternating paths with respect to their last dots. Alternating paths are essentially the zigzag paths defined by Corteel and Kim [6]. More precisely, a zigzag path starts with the west border of an unrestricted row, goes along a row and changes the direction when it comes across a topmost 1, then goes along a column and changes the direction when it meets a rightmost restricted 0 , and it ends at the southeast border.

It is worth mentioning that Steingrímsson and Williams [14] introduced a different kind of zigzag paths to establish a bijection between permutation tableaux and permutations. They defined a zigzag path as a path that starts with the northwest border of a permutation tableau, goes along the row or column until it reaches the southeast border, and changes the direction when it comes across a 1. Moreover, Burstein [2] defined a zigzag path as a path that changes the direction whenever it meets an essential 1.

It should be noted that an alternating path can be viewed as a path ending with the root in an alternative tree introduced by Nadeau [12]. However, in this paper, we shall define the inversion number directly on permutation tableaux without the formulation of alternative trees. It is straightforward to give an equivalent description in terms of alternative trees.

We conclude this paper with a connection between permutation tableaux without inversions and L-Bell tableaux introduced by Corteel and Nadeau [7].

\section{The inversion number of a permutation tableau}

In this section, we define the inversion number of a permutation tableau. We show that the inversion number of a permutation tableau $T$ equals the number of occurrences of the dashed pattern 32-1 in the reverse complement of the permutation $\pi$ corresponding to $T$ under the first bijection of Corteel and Nadeau [7].

Let $\pi=\pi_{1} \pi_{2} \cdots \pi_{n}$ be a permutation on $[n]$. Denote by $f_{\sigma}(\pi)$ the number of occurrences of a dashed pattern $\sigma$ in $\pi$. The reverse complement of $\pi$ is defined by

$$
\bar{\pi}=\left(n+1-\pi_{n}, \ldots, n+1-\pi_{2}, n+1-\pi_{1}\right),
$$

where a permutation is written in the form of a vector. 
Throughout this paper, we use $\Phi$ to denote the first bijection of Corteel and Nadeau [7] from permutation tableaux of length $n$ to permutations on $[n]$. The main result of this paper is the following relation.

Theorem 2.1. Let $T$ be a permutation tableau. Let inv $(T)$ be the number of inversions of $T$. Then we have

$$
\operatorname{inv}(T)=f_{32-1}(\bar{\pi})
$$

Since an occurrence of the dashed pattern $32-1$ in $\bar{\pi}$ corresponds to an occurrence of the dashed pattern $3-21$ in $\pi$, relation (2.1) can be restated as

$$
\operatorname{inv}(T)=f_{3-21}(\pi)
$$

To define the inversion number of a permutation tableau, we shall use the notion of zigzag paths of a permutation tableau defined by Corteel and Kim [6]. A zigzag path can be reformulated in terms of the alternative representation of a permutation tableau introduced by Corteel and Kim [6]. The alternative representation of a permutation tableau $T$ is obtained from $T$ by replacing the topmost 1's with $\uparrow$ 's, replacing the rightmost restricted 0's with $\leftarrow$ 's and leaving the remaining cells blank. It is not difficult to see that a permutation tableau can be recovered from its alternative representation. In this paper, we shall use black dots and white dots to represent the topmost 1's and the rightmost restricted 0's in an alternative representation. For example, the first tableau in Figure 2.1 is a permutation tableau of length 12 , and the second tableau gives the alternative representation and a zigzag path.

For the purpose of this paper, we shall use an equivalent description of zigzag paths by assuming that a zigzag path starts with a dot (either black or white) and goes northwest until it reaches the last black dot. To be more specific, for a white dot we can find a black dot strictly above as the next dot. For a black dot which is not in an unrestricted row, define the unique white dot on the left as the next dot. Such paths are called alternating paths. For example, in Figure 2.1, the third diagram exhibits two alternating paths.

It is easily seen that an alternating path can be represented as an alternating sequence of row and column labels ending with a column label of a black dot in an unrestricted row, since a black dot is determined by a column label and a white dot is determined by a row label. For example, for the black dot in cell $(5,6)$, the alternating path is $(6,5,12)$. For the white dot in cell $(7,10)$, the corresponding alternating path is $(7,10,4,11)$.

To define the inversion number of a permutation tableau, we shall introduce a linear order on alternating paths. Given two alternating paths $P$ and $Q$ of $T$, we say that $P$ is contained in $Q$ if $P$ is a segment of $Q$. If an alternating path $P$ is strictly contained in $Q$, then we define $P>Q$.

When $P$ is not contained in $Q$ and $Q$ is not contained in $P$ either, we define the order of $P$ and $Q$ as follows. If $P$ and $Q$ intersect at some dot, then they will share the same ending segment after this dot. If this is the case, we will remove the common dots of $P$ and $Q$, and then consider the resulting alternating paths $P^{\prime}$ and $Q^{\prime}$. Let $p_{e}$ (or $p_{e}^{\prime}$ ) denote the last dot of the path $P$ (or $P^{\prime}$ ) and let $q_{e}$ (or $q_{e}^{\prime}$ ) denote the last dot of the path $Q$ (or $\left.Q^{\prime}\right)$. We say that $P>Q$ if one of the following two conditions holds: 


\begin{tabular}{|c|c|c|c|c|c|}
\hline 0 & 1 & 0 & 0 & 0 & 1 \\
\hline 1 & 1 & 0 & 0 & 0 & $3^{2}$ \\
\hline 0 & 0 & 1 & 1 & 0 & 4 \\
\hline 0 & 1 & 1 & 1 & 1 & 5 \\
\hline 0 & 0 & 0 & 1 & $7^{6}$ & \\
\hline 0 & 0 & 0 & $9^{8}$ & & \\
\hline
\end{tabular}
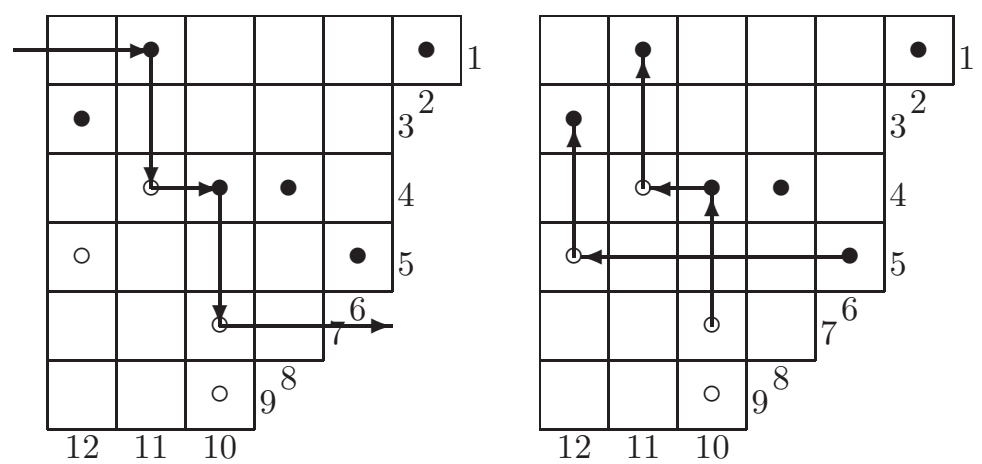

Figure 2.1: A permutation tableau, a zigzag path and two alternating paths.

(1) The last dots $p_{e}$ (resp. $\left.p_{e}^{\prime}\right)$ and $q_{e}$ (resp. $q_{e}^{\prime}$ ) are in the same row, and the last dot $p_{e}\left(\operatorname{resp} . p_{e}^{\prime}\right)$ is to the right of $q_{e}\left(\operatorname{resp} . q_{e}^{\prime}\right)$.

(2) The last dots $p_{e}$ (resp. $\left.p_{e}^{\prime}\right)$ and $q_{e}\left(\right.$ resp. $q_{e}^{\prime}$ ) are not in the same row, then the last dot of $p_{e}\left(\right.$ resp. $\left.p_{e}^{\prime}\right)$ is below $q_{e}\left(\right.$ resp. $\left.q_{e}^{\prime}\right)$.
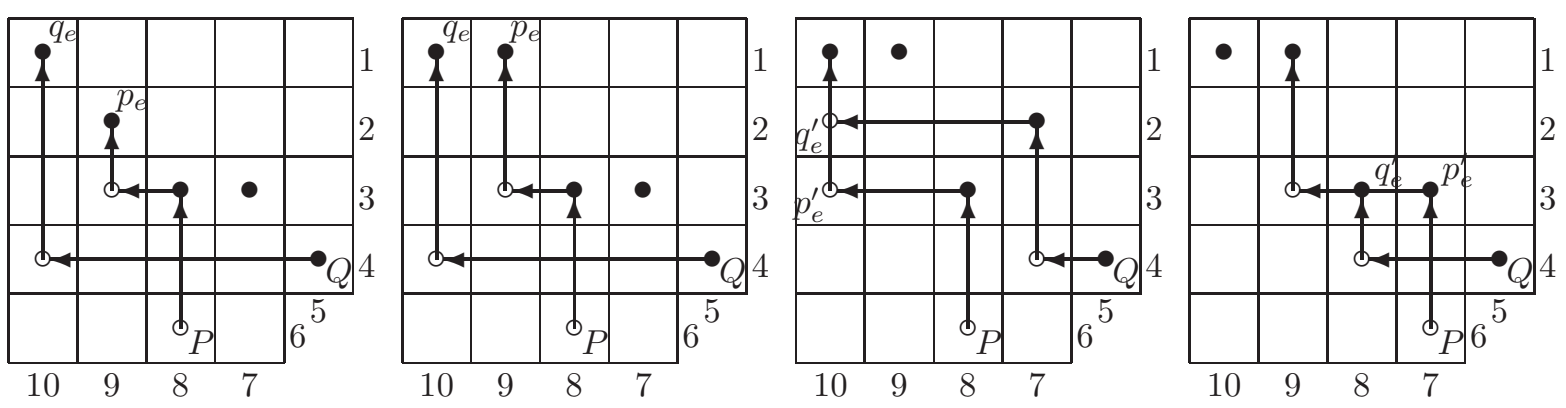

Figure 2.2: The cases for $P>Q$.

As shown in Figure 2.2, for any two alternating paths $P$ and $Q$ for which one is not contained in the other, there are four cases for the relation $P>Q$ to hold. It can be seen that for any distinct alternating paths $P$ and $Q$, we have either $P>Q$ or $Q>P$. Using this order, we can define the inversion number of an alternative representation $T$ of a permutation tableau. We shall consider the inversion number of the alternative representation as the inversion number of the original permutation tableau. Notice that it is easy to reformulate the definition of the inversion number of a permutation tableau in terms of the corresponding alternative tree introduced by Nadeau [12].

Definition 2.2. Suppose that $j$ is a column label of $T$ and $P_{j}$ is the alternating path starting with the black dot with column label $j$. Let $k$ be a label of $T$ with $j<k$ and let $P_{k}$ denote the alternating path starting with the dot labeled by $k$. We say that the pair of labels $(j, k)$ is an inversion of $T$ if $P_{j}>P_{k}$. The total number of inversions of $T$ is denoted by $\operatorname{inv}(T)$. 
For a column label $j$, we define $w_{j}(T)$ to be the number of inversions of $T$ that are of the form $(j, k)$. Hence

$$
\operatorname{inv}(T)=\sum_{j \in C(T)} w_{j}(T),
$$

where $C(T)$ is the set of column labels of $T$.

For example, Figure 2.3 gives two permutation tableaux in the form of their alternative representations. For the alternative representation $T$ on the left, we have $C(T)=\{2,3\}$. Since $P_{2}>P_{3}$, we see that $w_{2}(T)=1, w_{3}(T)=0$, and $\operatorname{inv}(T)=1$. For the alternative representation $T^{\prime}$ on the right, we have $C\left(T^{\prime}\right)=\{3,5\}$. Since $P_{3}>P_{4}$ and $P_{3}>P_{5}$, we find $w_{3}\left(T^{\prime}\right)=2, w_{5}\left(T^{\prime}\right)=0$, and $\operatorname{inv}\left(T^{\prime}\right)=2$.
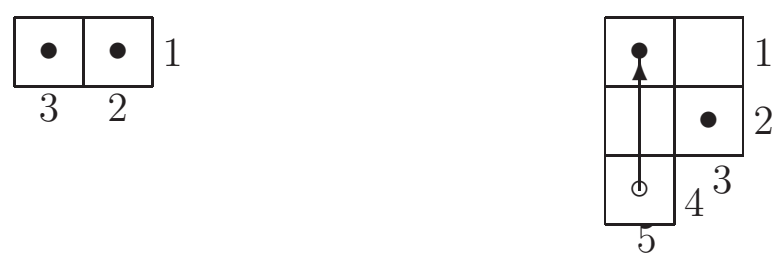

Figure 2.3: Two examples.

To present the proof of Theorem 2.1, we need to give an overview of the bijection $\Phi$ of Corteel and Nadeau from permutation tableaux to permutations. Assume that $T$ is the alternative representation of a permutation tableau. Let $\Phi(T)=\pi=\pi_{1} \pi_{2} \cdots \pi_{n}$. The bijection can be described as a recursive procedure to construct $\pi$. Starting with the sequence of the labels of unrestricted rows in increasing order. Then successively insert the column labels of $T$. Let $j$ be the maximum column label to be inserted. If cell $(i, j)$ is filled with a black dot, then insert $j$ immediately to the left of $i$. If column $j$ contains white dots in rows $i_{1}, i_{2}, \ldots, i_{k}$, then insert $i_{1}, i_{2}, \ldots, i_{k}$ in increasing order to the left of $j$. Repeating this process, we obtain a permutation $\pi$.

For example, let $T$ be the permutation tableau given in Figure 2.1. Then we have

$$
\Phi(T)=(7,9,10,8,4,11,2,1,6,5,12,3) .
$$

The following lemmas will be used in the proof of Theorem 2.1. The first was observed by Corteel and Nadeau [7].

Lemma 2.3. Let $\pi=\Phi(T)$. Then $\pi_{i}>\pi_{i+1}$ if and only if $\pi_{i}$ is a column label of $T$.

The next lemma states that the labels representing an alternating path of $T$ form a subsequence of $\Phi(T)$.

Lemma 2.4. Let $P=p_{1} p_{2} \cdots p_{r}$ be an alternating path of $T$ starting with a dot labeled by $p_{1}$ and ending with a black dot labeled by $p_{r}$. Then $p_{1} p_{2} \cdots p_{r}$ is a subsequence of $\Phi(T)$. 
Proof. Assume that the alternating path $P$ ends with a black dot at cell $\left(i, p_{r}\right)$, where $i$ is an unrestricted row label. Since the last dot represents a topmost 1, by the construction of $\Phi$, we see that $p_{r}$ is inserted to the left of $i$. Note that cell $\left(p_{r-1}, p_{r}\right)$ is filled with a white dot representing a rightmost restricted 0 , so $p_{r-2}$ is inserted to the left of $p_{r-1}$. Since the path $P$ is alternating with respect to black and white dots, we deduce that the elements $p_{r-3}, \ldots, p_{2}, p_{1}$ are inserted one after another such that $p_{i}$ is inserted to the left of $p_{i+1}$ for $i=1,2, \ldots, r-1$. It follows that $p_{1} p_{2} \cdots p_{r}$ is a subsequence of the permutation $\Phi(T)$. This completes the proof.

Given two labels $i$ and $j$ of $T$, the following lemma shows that the relative order of $i$ and $j$ in $\Phi(T)$ can be determined by the order of the alternating paths starting with the dots labeled by $i$ and $j$.

Lemma 2.5. Let $P_{i}$ and $P_{j}$ be two alternating paths of $T$ starting with two dots labeled by $i$ and $j$. Then $i$ is to the left of $j$ in $\Phi(T)$ if and only if $P_{j}>P_{i}$.

Proof. First, we show that if $P_{j}>P_{i}$, then $i$ is to the left of $j$ in $\Phi(T)$. When $P_{j}$ is contained in $P_{i}$, by Lemma 2.4, we see that $i$ is to the left of $j$. We now turn to the case when $P_{j}$ is not contained in $P_{i}$. In this case, let $P_{i}=i_{1} i_{2} \cdots i_{s}$ and $P_{j}=j_{1} j_{2} \cdots j_{t}$, where $i=i_{1}$ and $j=j_{1}$.

If $P_{i}$ and $P_{j}$ do not intersect, by Lemma 2.4, we see that $i_{1}$ is to the left of $i_{s}$. So it suffices to show that $j_{1}$ is to the right of $i_{s}$ in $\Phi(T)$. Suppose that the last black dots of $P_{i}$ and $P_{j}$ are in cells $\left(r_{P_{i}}, i_{s}\right)$ and $\left(r_{P_{j}}, j_{t}\right)$ respectively, where $r_{P_{i}}$ and $r_{P_{j}}$ are the labels of unrestricted rows. By definition, we have either $r_{P_{i}}<r_{P_{j}}$ or $r_{P_{i}}=r_{P_{j}}$. Thus we have two cases.

Case 1: $r_{P_{i}}<r_{P_{j}}$. By the construction of $\Phi, r_{P_{i}}$ is to the left of $r_{P_{j}}$ in $\Phi(T)$. Since both cells $\left(r_{P_{i}}, i_{s}\right)$ and $\left(r_{P_{j}}, j_{t}\right)$ are filled with black dots, the element $i_{s}$ is inserted immediately to the left of $r_{P_{i}}$, while $j_{t}$ is inserted immediately to the left of $r_{P_{j}}$. This implies that $j_{t}$ is to the right of $r_{P_{i}}$ in $\Phi(T)$. Hence $j_{t}$ is to the right of $i_{s}$.

Since $P_{j}$ is an alternating path consisting of black and white dots, cell $\left(j_{t-1}, j_{t}\right)$ is filled with a white dot. Thus $j_{t-1}$ is inserted to the left of $j_{t}$ but to the right of $r_{P_{i}}$, that is, $j_{t-1}$ is to the right of $i_{s}$. Iterating the above procedure, we reach the conclusion that the label $j_{r}$ is to the right of $i_{s}$ for $r=t, t-1, \ldots, 1$. In particular, $j_{1}$ is to the right of $i_{s}$, so that $j_{1}$ is to the right of $i_{1}$.

Case 2: $r_{P_{i}}=r_{P_{j}}$. Since $P_{j}>P_{i}$, we have $j_{t}<i_{s}$. In the implementation of the algorithm $\Phi, i_{s}$ is inserted immediately to the left of $r_{P_{i}}$ and then $j_{t}$ is inserted immediately to the left of $r_{P_{j}}=r_{P_{i}}$. Hence $j_{t}$ is to the right of $i_{s}$. Inspecting the relative positions of $i_{s}$ and $j_{r}$ for $r<t$ as in Case 1, we see that $j_{r}$ is to the right of $i_{s}$ for $r=t, t-1, \ldots, 1$. So we arrive at the conclusion that $j_{1}$ is to the right of $i_{1}$.

It remains to consider the case when $P_{i}$ intersects $P_{j}$. As shown before, in this case, $P_{i}$ and $P_{j}$ have a common ending segment starting from the intersecting dot. Let $P_{i}^{\prime}$ and $P_{j}^{\prime}$ be the alternating paths obtained by removing the common segment of $P_{i}$ and $P_{j}$. Suppose that the last dot of $P_{i}^{\prime}$ is labeled by $i_{s-m}$. It can be seen that the last dot of $P_{j}^{\prime}$ is labeled by $j_{t-m}$. By Lemma 2.4, $i_{1}$ is to the left of $i_{s-m}$. 
We now aim to show that $j_{1}$ is to the right of $i_{s-m}$ in $\Phi(T)$. We have the following two cases.

Case A: The last dot of $P_{j}^{\prime}$ is below the last dot of $P_{i}^{\prime}$, that is, $j_{t-m}>i_{s-m}$. In this case, both cells $\left(i_{s-m}, i_{s-m+1}\right)$ and $\left(j_{t-m}, j_{t-m+1}\right)$ are filled with white dots. To construct $\pi$ from $T$ by using the bijection $\Phi$, both elements $i_{s-m}$ and $j_{t-m}$ are inserted to the left of the element $i_{s-m+1}=j_{t-m+1}$ in increasing order. Hence $i_{s-m}$ is to the left of $j_{t-m}$. Considering the relative positions of $i_{s-m}$ and $j_{r}$ for $r<t-m$ as in Case 1, we deduce that $j_{r}$ is to the right of $i_{s-m}$ for $1 \leq r<t-m$. Therefore $j_{1}$ is to the right of $i_{s-m}$, and hence to the right of $i_{1}$.

Case B: The last dot of $P_{j}^{\prime}$ is to the right of the last dot of $P_{i}^{\prime}$, that is, $j_{t-m}<i_{s-m}$. Observe that the element $i_{s-m}$ is inserted immediately to the left of $i_{s-m+1}$. Moreover, the element $j_{t-m}$ is inserted immediately to the left of $j_{t-m+1}=i_{s-m+1}$. It follows that $j_{t-m}$ is to the right of $i_{s-m}$. Applying the same argument as in Case 1 to elements $i_{s-m}$ and $j_{r}$ for $r<t-m$, we conclude that $j_{r}$ is to the right of $i_{s-m}$. Consequently, $j_{1}$ is to the right of $i_{s-m}$, and hence to the right of $i_{1}$.

In summary, we deduce that if $P_{j}>P_{i}$, then $i$ is to the left of $j$ in $\Phi(T)$.

Finally, we need to show that if $i$ is to the left of $j$ in $\Phi(T)$, then we have $P_{j}>P_{i}$. Assume that $i$ is to the left of $j$ in $\Phi(T)$. Consider the order of $P_{i}$ and $P_{j}$. Clearly, we have $P_{i} \neq P_{j}$, that is, we have either $P_{j}>P_{i}$ or $P_{i}>P_{j}$. If $P_{i}>P_{j}$, as shown before, we see that $j$ is to the left of $i$ in $\Phi(T)$. Thus we only have the case $P_{j}>P_{i}$. This completes the proof.

We are now ready to prove the main theorem.

Proof of Theorem 2.1. Let $\Phi(T)=\pi=\pi_{1} \pi_{2} \cdots \pi_{n}$. Combining Lemma 2.3 and Lemma 2.5, we find that the subsequence $\pi_{i} \pi_{j} \pi_{j+1}$ of $\pi$ is an occurrence of the dashed pattern 3-21 if and only if $\left(\pi_{j}, \pi_{i}\right)$ is an inversion of $T$. It follows that

$$
\operatorname{inv}(T)=f_{3-21}(\pi),
$$

as desired. This completes the proof.

Let us give an example of Theorem 2.1. Let $T$ be the alternative representation of the permutation tableau given in Figure 2.4.

\begin{tabular}{|c|c|c|c|}
\hline 1 & 1 & 0 & 0 \\
\hline 0 & 0 & 0 & 0 \\
\hline 1 & 1 & 1 & 1 \\
\hline 1 & 1 & 1 & 1 \\
\hline 0 & 0 & $7^{6}$ & 5 \\
\hline
\end{tabular}

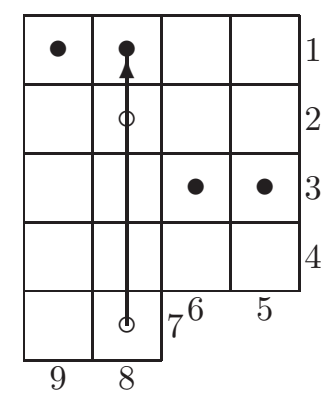

Figure 2.4: A permutation tableau and its alternative representation.

We see that $C(T)=\{5,6,8,9\}, w_{5}(T)=4, w_{6}(T)=3, w_{8}(T)=1$ and $w_{9}(T)=0$. 
Hence we have $\operatorname{inv}(T)=8$. On the other hand,

$$
\pi=\Phi(T)=(9,2,7,8,1,6,5,3,4) .
$$

So we have $\bar{\pi}=(6,7,5,4,9,2,3,8,1)$. It can be checked that the number of occurrences of the dashed pattern $32-1$ in $\bar{\pi}$ is 8 .

\section{Connection to L-Bell tableaux}

In this section, we show that a permutation tableau has no inversions if and only if it is an L-Bell tableau as introduced by Corteel and Nadeau [7]. Recall that an L-Bell tableau is a permutation tableau such that any topmost 1 is also a leftmost 1 .

It has been shown by Claesson [3] that the number of permutations on $[n]$ avoiding the dashed pattern $32-1$ is given by the $n$-th Bell number $B_{n}$. In view of Theorem 2.1, we are led to the following correspondence.

Theorem 3.1. The number of permutation tableaux $T$ of length $n$ such that inv $(T)=0$ equals $B_{n}$.

On the other hand, the following relation was proved by Corteel and Nadeau [7].

Theorem 3.2. The number of L-Bell permutation tableaux of length $n$ equals $B_{n}$.

By the definition of an inversion of a permutation tableau, it is straightforward to check that an L-Bell tableau has no inversions. Combining Theorem 3.1 and Theorem 3.2 , we obtain the following relation.

Theorem 3.3. Let $T$ be a permutation tableau. Then inv $(T)=0$ if and only if $T$ is an L-Bell tableau.

Here we give a direct reasoning of the above theorem. Let $T$ be an alternative representation of a permutation tableau without inversions. It can be seen that the permutation tableau corresponding to $T$ is an L-Bell tableau if and only if $T$ satisfies the following conditions:

(1) Each row contains at most one black dot.

(2) If there is an empty cell such that there is a black dot to the right and there is no white dot in between, then all the cells above this empty cell are also empty.

We wish to prove that if $\operatorname{inv}(T)=0$, then $T$ satisfies the above conditions.

Assume that there is a row containing two black dots, say, at cells $(i, j)$ and $(i, k)$ with $j<k$. Clearly, by definition, $(j, k)$ is an inversion of $T$. So we are led to a contradiction. This implies that condition (1) holds.

With respect to condition (2), we may assume to the contrary that there exists a dot above some empty cell $(i, k)$ and relative to this empty cell, there is a black dot to the 
right and there is no white dot between cell $(i, k)$ and the black dot to the right. Without loss of generality, we may assume that $i$ is the minimal row label of an empty cell subject to the above assumption. We may choose $k$ to be the maximal column label. Assume that the black dot in row $i$ and the black dot in column $k$ are located at cells $(i, j)$ and $(t, k)$. Evidently, we have $t<i$. Since $j<k$ and $\operatorname{inv}(T)=0$, we see that $P_{j}>P_{k}$. This implies that the unrestricted row containing the last black dot of $P_{j}$ must be above row $t$.

Assume that the last black dot is in column $m$. Since the black dot at cell $(t, k)$ is not the last dot (otherwise, $(k, m)$ is an inversion of $T$, a contradiction to the assumption that $\operatorname{inv}(T)=0)$. So we deduce that there exists a white dot in row $t$. Suppose that it occurs at cell $(t, s)$. Since any cell $(x, y)$ must be empty for $x<t, k<y<s$, we find that $s \leq m$; otherwise, cell $(t, m)$ would be an empty cell satisfying condition (2) but with a row label smaller than $i$, contradicting the choice of cell $(i, k)$. Moreover, for $x>t$ and $y>s$, any cell $(x, y)$ cannot be filled with a dot which is on the path $P_{j}$. Otherwise, there exists a row $l$ containing a white dot to the left of column $s$ and a black dot to the right of column $s$. Thus, the empty cell $(l, s)$ satisfies condition $(2)$ but with a row label smaller than $i$, a contradiction to the choice of cell $(i, k)$.

By walking backwards starting from the last black dot $p$ along the path $P_{j}$, we shall meet a black dot in column $s$ on the alternating paths $P_{j}$ and $P_{k}$. Figure 3.1 gives an illustration showing that the paths $P_{j}$ and $P_{k}$ intersect at column $s$. Note that any cell in area $A$ must be empty and the cells in area $B$ cannot be filled with any dot that is on the path $P_{j}$.

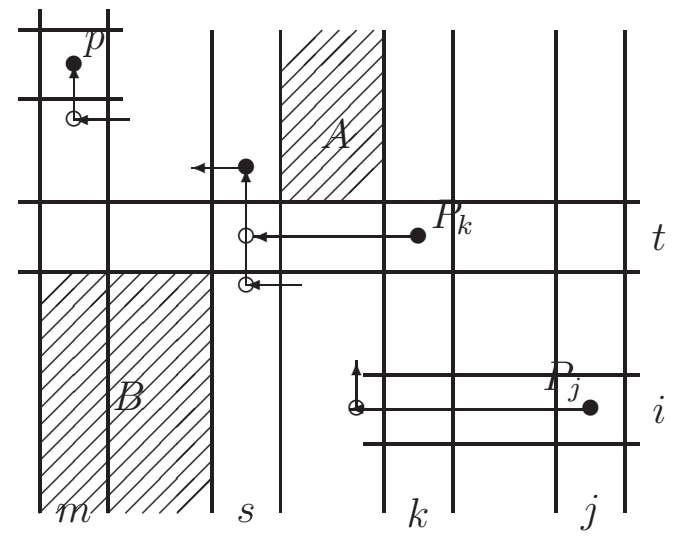

Figure 3.1: An illustration.

So we conclude that the next white dot on $P_{j}$ is below the next white $\operatorname{dot}$ on $P_{k}$, that is, $P_{j}>P_{k}$. But this implies that $(j, k)$ is an inversion of $T$, again a contradiction. Hence the proof is complete.

Acknowledgments. We are grateful to the referees for helpful suggestions. This work was supported by the 973 Project, the PCSIRT Project of the Ministry of Education, and the National Science Foundation of China. 


\section{References}

[1] E. Babson and E. Steingrímsson, Generalized permutation patterns and a classification of the Mahonian statistics, Sém. Lothar. Combin. 44 (2000), B44b.

[2] A. Burstein, On some properties of permutation tableaux, Ann. Combin. 11 (2007), $355-368$.

[3] A. Claesson, Generalized pattern avoidance, European J. Combin. 22 (2001), 961971.

[4] A. Claesson and T. Mansour, Counting occurrences of a pattern of type $(1,2)$ or $(2$, 1) in permutations, Adv. in Appl. Math. 29 (2002), 293-310.

[5] S. Corteel, R. Brak, A. Rechnitzer and J. Essam, A combinatorial derivation of the PASEP stationary state, Electron. J. Combin 13 (2006), \#R108.

[6] S. Corteel and J. S. Kim, Combinatorics on permutation tableaux of type $A$ and type B, European J. Combin. 32 (2011), 563-579.

[7] S. Corteel and P. Nadeau, Bijections for permutation tableaux, European J. Combin. 30 (2009), 295-310.

[8] S. Corteel and L. Williams, Tableaux combinatorics for the asymmetric exclusion process I, Adv. in Appl. Math. 37 (2007), 293-310.

[9] S. Corteel and L. Williams, Tableaux combinatorics for the asymmetric exclusion process and Askey-Wilson polynomials, arXiv:math.CO/0910.1858.

[10] S. Corteel and L. Williams, A Markov chain on permutations which projects to the PASEP, Int. Math. Res. Not. (2007), Art. ID rnm055.

[11] T. Lam and L. Williams, Total positivity for cominuscule Grassmannians, New York J. Math. 14 (2008), 53-99.

[12] P. Nadeau, The structure of alternative tableaux, J. Combin. Theory Ser. A 118 (2011), 1638-1660.

[13] A. Postnikov, Total positivity, Grassmannians, and networks, arXiv:math/0609764.

[14] E. Steingrímsson and L. Williams, Permutation tableaux and permutation patterns, J. Combin. Theory Ser. A 114 (2007), 211-234.

[15] X. Viennot, Alternative tableaux, permutations and partially asymmetric exclusion process, Isaac Newton Institute, April 2007.

[16] L. Williams, Enumeration of totally positive Grassmann cells, Adv. in Math. 190 (2005), 319-342. 